Rabaska

Revue d'ethnologie de l'Amérique française

\title{
Dévoiler la couleur des textiles domestiques : rencontre de la tradition orale et de l'analyse scientifique
}

\section{Louise Lalonger}

Volume 13, 2015

Présence de Marius Barbeau : l'invention du terrain en Amérique

française. Autour d'un legs centenaire (1914-2014)

URI : https://id.erudit.org/iderudit/1033763ar

DOI : https://doi.org/10.7202/1033763ar

Aller au sommaire du numéro

Éditeur(s)

Société québécoise d'ethnologie

ISSN

1703-7433 (imprimé)

1916-7350 (numérique)

Découvrir la revue

Citer cet article

Lalonger, L. (2015). Dévoiler la couleur des textiles domestiques : rencontre de la tradition orale et de l'analyse scientifique. Rabaska, 13, 209-217.

https://doi.org/10.7202/1033763ar 


\title{
Dévoiler la couleur des textiles domestiques : rencontre de la tradition orale et de l'analyse scientifique
}

\author{
LOUISE LALONGER \\ Restauratrice de biens culturels \\ Centre de conservation du Québec
}

En 1856, William Henry Perkin découvre le premier colorant synthétique, la mauvéine ${ }^{1}$. Âgé d'à peine 18 ans à ce moment-là, il fait cette découverte par hasard alors qu'il s'efforce de trouver un procédé pour synthétiser la quinine à partir de la houille de charbon. Il est loin de se douter de l'impact de sa découverte qui viendra transformer un savoir-faire millénaire, où les couleurs sont tirées uniquement de la nature. Grâce à la découverte de Perkin, l'Angleterre sera la première à commercialiser les colorants synthétiques, suivie de près par la France et l'Allemagne.

Le succès considérable de la mauvéine incite ensuite de nombreux chimistes à entreprendre des recherches analogues. C'est ainsi que plusieurs dizaines de colorants synthétiques sont mis au point pendant les années qui suivent. Les premières découvertes apparaissent petit à petit entre 1856 et 1869 avec l'élaboration de produits donnant principalement le bleu, le rouge et le violet. La décennie suivante voit naître de nombreux tons de jaune, d'orangé et de rouge grâce à l'invention de l'alizarine synthétique (Graebe et Liebermann 1868, Perkin 1869$)^{2}$ qui présente les mêmes propriétés que la garance naturelle. Quoique les années 1880 offrent une gamme de colorants de synthèse de plus en plus variée, certaines teintures naturelles persistent, tel l'indigo, qui n'est synthétisé et mis en marché qu'en $1897^{3}$. C'est ainsi que l'indigo naturel est encore utilisé en cette fin du XIX ${ }^{e}$ siècle et en début du XIX siècle, soit jusqu'à 1920 environ.

1. Rita-J. Adrosko, Natural Dyes in United-States, Washington, Smithsonian Institution Press, 1968, p. 3.

2. Mary W. Ballard et al., Important Early Synthetic Dyes : chemistry, constitution, date, propreties, Washington, Conservation Analytical Laboratory, Smithsonian Institution, 1989, n. p.

3. George F. Jaubert, La Garance et l'indigo, Paris, Encyclopédie scientifique des aide-mémoire, n. d., p. 74 . 
En observant cette période de transition, il est fort à propos de se demander de quelle manière s'est effectué le passage des colorants naturels aux colorants synthétiques et dans quelle mesure l'on retrouve ces nouveaux produits pour la fabrication de textiles domestiques en milieu rural. Nous verrons ce sujet sous deux angles : d'abord avec le regard de l'ethnologue, qui appuie sa recherche sur des données recueillies sur le terrain, puis avec le regard du chimiste, qui fonde l'identification des colorants à partir de tests réalisés en laboratoire.

\section{Les enquêtes ethnographiques de Marius Barbeau}

Les premières mentions de plantes tinctoriales en Nouvelle-France nous viennent du voyageur Pehr $\mathrm{Kalm}^{4}$ au XVIII ${ }^{\mathrm{e}}$ siècle, un botaniste suédois qui a fait une étude d'histoire naturelle d'Amérique du Nord. Les Français du Canada apprennent des Amérindiens l'usage du gaillet des teinturiers, appelé « tisavoyanne rouge », de même que celui de la « tisavoyanne jaune », soit le coptide du Groënland que Kalm appelle « ellébore à trois feuilles ». Ce dernier observe également des femmes de Montmorency qui teignent en jaune avec des graines du myrique baumier qu'il nomme « myrte bâtard ou poivrier ».

La principale source d'enquêtes orales sur les teintures avec des colorants naturels nous provient du fonds Marius Barbeau des archives du Musée canadien de l'histoire ${ }^{5}$. Un dossier sur le sujet regroupe quelques pages de carnets d'enquêtes découpées. Les notes de Marius Barbeau avaient été prises avec la sténographie qui lui est propre, transcrites et dactylographiées. Le fait que les pages soient découpées nous fait perdre certaines données, dont le contexte de l'enquête. Par contre, on a toujours le nom de l'informateur et la municipalité.

Ces enquêtes ont été réalisées auprès d'une trentaine d'informateurs, qui proviennent principalement du comté de Charlevoix, mais également de l'Île-d'Orléans, de Notre-Dame-du-Portage, du Mont-Louis en Gaspésie et de la Jeune-Lorette (aujourd'hui Wendake). Seules deux pages sont datées : on sait que Barbeau enquêtait à la Jeune-Lorette en 1911 et à Notre-Damedu-Portage en 1918. Néanmoins, on trouve les noms des autres informateurs dans d'autres sources (liste d'achat, fonds photographique), ce qui nous permet de croire que Barbeau les a rencontrés entre 1930 et 1938.

Les fautes d'orthographe, qui ont pu se glisser lors de la transcription des notes de Barbeau, rendent l'interprétation difficile. Par exemple, le mot « couperose », qui est le nom ancien pour les sulfates de cuivre (CuSO4) ou

4. Pierre Kalm, Voyages de Kalm en Amérique, traduit par L.W. Marchand, Montréal, Mémoires de la Société historique de Montréal, 1880, 2 vol.

5. Boîte 147 F1. 
de fer (FeSO4), est bien connu comme mordant ${ }^{6}$ pour la teinture. Dans les transcriptions, ce mot se retrouve orthographié comme suit : " conperose ", « conperosa », « conpense », « compérose », etc. Ces erreurs rendent difficile l'identification botanique des plantes à partir des noms transcrits avec différentes orthographes. Ainsi, le nom vernaculaire du sorbier d'Amérique, le " Masquabina », devient " Masquatrine ", " Maskabruna " ou « Masquamina ». Dans ce cas-ci, on ne peut distinguer s'il s'agit de noms différents d'un informateur à l'autre ou simplement d'une erreur de transcription.

Le dépouillement des enquêtes orales de Barbeau a fait ressortir que les artisanes de la vallée du Saint-Laurent connaissaient des techniques de teinture végétale. Ces gens auraient employé des matières colorantes déjà utilisées traditionnellement en Europe depuis fort longtemps, entre autres l'oignon, l'aulne, le bouleau et le thé noir. La teinture avec le sumac vinaigrier est connue depuis l'Antiquité grecque et égyptienne ${ }^{7}$, alors que certaines sont en usage depuis le Moyen Âge comme la verge d'or et l'écorce de pommier8. D'autres produits tinctoriaux illustrent l'adaptation des colons français à leur environnement avec l'usage plus régionalisé de plantes indigènes comme le kalmia, le coptide du Groenland et le lédon du Groenland, ou encore le sorbier d'Amérique et le tsuga du Canada (ou pruche).

Les enquêtes démontrent que l'usage des colorants naturels importés était connu. Une dizaine d'informateurs se rappellent de l'indigo, dont cet informateur de Baie-Saint-Paul qui décrit la cuve à l'indigo par fermentation à l'urine. Il nous dit :

On ramassait l'urine une journée d'avance. On mettait à tremper l'indigo dans un petit sac encore une journée... on mettait une once par jour dans le petit sac... Tous les matins, il fallait tordre la laine qui était dans la teinture et l'urine. La laine trempait comme ça huit jours... Il fallait pour ça se boucher la bouche et le nez avec une bande pour y faire tourner la teinture. Il ne fallait pas respirer.

De plus, une informatrice de Miscoutine nous démontre la grande qualité de cette teinture : " L'indigo fait de la belle teinture. C'est malaisé à teindre, mais l'indigo ne se déteint pas, ne change pas. Toutes les teintures qu'on achète ça déteint. »

Viennent ensuite les bois tinctoriaux exotiques, comme le bois rouge (bois du Brésil), le bois jaune (mûrier des teinturiers) et le bois noir (bois de campêche). Une informatrice des Éboulements précise : «Autrefois on achetait du bois à la livre. C'était tout par petits morceaux et on teignait avec ça en rouge. Ça teignait, ça changeait point. On achetait ça chez le marchand. On

6. En teinture, le terme « mordant » désigne un agent intermédiaire (souvent un sel métallique) qui aide à fixer le colorant ou sert à modifier la couleur.

7. Dominique Cardon, Guide des teintures naturelles, Paris, Delachaux et Niestlé, 1990, p. 274.

8. Ibid., p. 71 et 289. 
descendait ça de Québec [...]». En 1918, un informateur de Notre-Dame-duPortage nous donne une indication sur la période d'utilisation de ces colorants, qu'il nomme «bois des îles », en signalant qu'ils « étaient employés encore il y a une trentaine d'années » (soit vers 1880). La popularité de ces bois tinctoriaux se reflète dans une publicité de 1873 de l'Apothicairerie du peuple à Québec, qui faisait la promotion des teintures domestiques et capillaires sous le nom de « Bois noir, rouge et jaune $»^{9}$.

Sept informateurs ont mentionné l'usage de teintures « achetées », que nous pouvons associer aux colorants synthétiques. Les couleurs relevées dans l'enquête sont le magenta, l'orangé, le rouge et le vert. Les propos d'un informateur des Éboulements indiquent à quel point il apprécie la vivacité de ces nouvelles couleurs. Il décrit une couverture « boutonnue» de sa famille. Elle était ornée de motifs d'arbres, des amandes, des denticules. Il identifie les couleurs : la teinture indigo, le rouge dindon et le vert, " un beau vert bouteille acheté ». Il poursuit à propos de la teinture naturelle au « bois de savane » qui donnait des jaunes et des bruns : « Ça ne fait pas de couleurs éclatantes comme la teinture qu'on achète $»$.

Marius Barbeau a décrit également la technique de réserve par ligature (ou ikat). Une informatrice de Sainte-Agnès se rappelle : " On prenait un écheveau sur le dévidoir, on prenait du fil et on l'attachait cordé bien dur pour empêcher l'eau chaude de traverser et on teignait ça en bleu ou autrement. Y avait des bouts teints et d'autres bouts qui ne l'étaient pas. On tricotait ça et ça donnait des beaux bas fleuris...»

L'autarcie des régions rurales nous laisse croire que la pratique de filer et de teindre sa laine était courante jusqu'au début du $\mathrm{xx}^{\mathrm{e}}$ siècle. Selon un rapport du ministère du Commerce fédéral, en 1926, on filait encore la laine pour un usage domestique dans $60 \%$ des maisons du Québec ${ }^{10}$.

\section{Témoins de la couleur : textiles sélectionnés et tests en laboratoire}

C'est le dépouillement d'enquêtes qui a permis de cibler les produits tinctoriaux les plus représentatifs d'une production domestique et de réaliser des échantillons de ces colorants. Nous avons réalisé des teintures avec quatorze plantes indigènes mentionnées dans les enquêtes, en utilisant cinq mordants métalliques ${ }^{11}$ qui en variaient la couleur. Nous sommes en mesure de constater les limites de la gamme de couleurs obtenues avec des plantes indigènes qui comprend des nuances de beige, de jaune, d'orangé, de vert, de brun et de gris. Nous pouvons dès lors mieux comprendre l'importance des colorants

9. Annuaire du commerce et de l'industrie de Québec, Québec, 1873, p. 32.

10. Henri Turcot, La Petite Industrie de la laine au Canada français, Ottawa, ministère du Commerce, 1928, p. 7.

11. Alun, chlorure d'étain, bichromate de potassium (ou chrome), sulfate de cuivre, sulfate de fer. 
importés (ou exotiques) qui complètent cette gamme avec principalement le bleu, le rouge, le violet et le noir.

À la suite de cette recherche, nous avons constitué un corpus de textiles sur lesquels il serait possible de faire l'identification des colorants. Les pièces sélectionnées ${ }^{12}$ étaient des textiles de facture domestique pour lesquels on a utilisé des laines filées à la main et sans doute teintes à la maison. Nous avons porté une attention particulière aux textiles attribués à la fin du XIX $X^{e}$ siècle et à la région de Charlevoix, ou du moins aux deux rives du Saint-Laurent. De ce corpus, nous avons prélevé quarante-trois échantillons, qui représentent neuf couleurs. Signalons que les couleurs prédominantes de ces échantillons sont le rouge, l'orangé et le violet.

L'identification des colorants sur les textiles sélectionnés nous amène à faire des tests chimiques en laboratoire, à partir de petits échantillons prélevés au dos des textiles, avec le consentement des musées. Préalablement, il nous a fallu constituer une banque d'échantillons témoins qui ont servi d'éléments de comparaison lors des tests. Cette collection de référence est constituée des principaux colorants synthétiques créés entre 1856 et 1902, des colorants naturels importés et des colorants indigènes mentionnés dans les enquêtes orales.

L'analyse chimique des colorants vise à déterminer s'il s'agit d'une teinture naturelle ou synthétique et à l'identifier spécifiquement. Les méthodes d'analyse retenues sont celles que préconise le chimiste Helmut Scheppe, un expert mondial sur le sujet. Ces méthodes d'analyse consistent principalement en des tests d'extraction et de chromatographie en couches minces (CCM). Ces techniques, bien que laborieuses, demandent peu d'équipements. Elles sont simples tout en étant précises et d'une grande sensibilité.

Nous avons également demandé la contre-expertise de laboratoires spécialisés qui disposent d'équipements de pointe. Quatre échantillons ont été envoyés au Textiles Research Associates en Angleterre. Chaque échantillon a été examiné par microscopie et a fait l'objet d'analyses par spectroscopie d'absorption et par chromatographie en couche mince (CCM).

De plus, nous avons fait appel au Service de la recherche analytique de l'Institut canadien de conservation (ICC), à Ottawa, pour quatre autres échantillons. Les extraits ont été analysés par chromatographie liquide à haute performance (CLHP) et par spectroscopie infrarouge à transformer de Fourier (SIRTF).

Ces tests ont permis de constater à quel point un colorant ne s'identifie pas à l'œil nu. C'est ainsi que quatre échantillons orangés, qui semblaient de même couleur que la teinture à la pelure d'oignon, présentaient pourtant

12. Les textiles sont tirés des collections suivantes: Musée québécois de culture populaire à Trois-Rivières, Parcs Canada à Québec et Musée de la civilisation à Québec. 
des produits synthétiques, soit les colorants Orange I (C.I. Acid Orange 20, $\mathrm{n}^{\circ} 14600$ ) et Orange II (C.I. Acid Orange 7, $\left.\mathrm{n}^{\circ} 15510\right)^{13}$.

De même, nous avons été étonnés d'identifier le violet de méthyle (C.I. Basic Violet 1, $\mathrm{n}^{\circ}$ 42535), tant sur un échantillon brun que sur un échantillon violet qui proviennent d'un même textile. Ici, ces deux couleurs de trame forment des rayures, en alternant irrégulièrement le brun et le violet. La teinture a été réalisée sur une fibre naturellement brune pour foncer la couleur. La décoloration de cette teinture ne laisse entrevoir que la couleur naturelle de la fibre.

Un échantillon rouge a été identifié comme étant une laque d'insecte, le Coccus lacca (Kerr, 1782). Il s'agit d'un insecte parasitaire provenant d'Asie qui vit dans une masse résineuse. Le colorant rouge est concentré dans le corps de l'insecte, dans les œufs et les larves.

\section{Résultats d'analyses : l'apport à la documentation et à la conservation-restauration}

L'utilisation de la laque à des fins domestiques paraît assez inusitée. En effet, aucune mention de ce colorant animal ne ressort de l'enquête orale ni des sources écrites. Ce produit a pu être importé au Québec par les Anglais qui commerçaient avec les Indes. Toutefois, nous ne pouvons préciser si ce produit nous est parvenu à l'état brut pour la teinture ou s'il s'agit d'une laine importée déjà teinte. Nous pouvons également entrevoir la possibilité d'une récupération d'un lainage rouge, cardé et filé à nouveau. Jean-Claude Dupont a d'ailleurs retracé cette façon de faire chez les Acadiens qui récupéraient des étoffes anglaises rouges qu'ils réduisaient en charpies, qu'ils cardaient et filaient pour les intégrer à leur tissage ${ }^{14}$. Cette pratique est connue également chez les Navajos aux États-Unis qui recyclaient de cette façon des draps de laine rouge (appelés bayeta) qu'on importait d'Angleterre ${ }^{15}$.

Les résultats d'analyses révèlent une prédominance des colorants synthétiques pour les textiles domestiques sélectionnés. Les seules exceptions concernent la laque d'insecte pour le rouge, et l'indigo pour le bleu que l'on retrouve combiné à des colorants synthétiques dans la composition des verts, des violets et des noirs. Avec ces nouvelles données, il est maintenant possible de corriger des erreurs d'attribution aux colorants naturels.

Pour trois textiles du corpus, nous arrivons à des conclusions différentes de l'information écrite. Bien qu'ils soient associés aux teintures végétales

13. Nous présentons les colorants avec la nomenclature internationale et les numéros du Color Index, Society of Dyers and Colourists, American Association of Textile Chemists and Colorists, $3^{e}$ éd. Bradford, 1971 (1956), vol. 1-4.

14. Jean-Claude Dupont, Histoire populaire de l'Acadie, Montréal, Leméac, 1979, p. 204.

15. J. R. Weissman, et W. Lavitt, Labors of Love : America's Textiles and Needlework, 1650-1930, New-York, Alfred Knopf, 1987, p. 214. 
dans l'inventaire des musées concernés, les analyses ont révélé qu'il s'agissait principalement de colorants synthétiques. Un seul de ces textiles présente l'indigo naturel en présence de colorants synthétiques, avec parfois la combinaison des deux. Cela vient confirmer l'importance des analyses chimiques avant de tirer des conclusions trop hâtives.

L'information sur les colorants synthétiques identifiés, leurs découvreurs et leurs dates de découverte apporte un éclaircissement sur la datation d'un textile. En confrontant les données de départ avec les nouvelles attributions, on note un écart d'environ vingt-cinq à cinquante ans. Ainsi, les textiles attribués au milieu du XIX ${ }^{\mathrm{e}}$ siècle sont maintenant datés de la toute fin de ce siècle (1880 à 1900). Ou encore, les textiles de la fin du XIX ${ }^{\mathrm{e}}$ sont maintenant associés au début $\mathrm{du} \mathrm{Xx}^{\mathrm{e}}$ siècle. Ces datations ne sont jamais très précises, mais tiennent compte de l'identification des colorants synthétiques et des informations à leur sujet. Nous sommes en mesure d'indiquer qu'ils sont postérieurs à une date donnée, en regard des dates de création des colorants identifiés.

Les analyses ont permis, en outre, d'observer et d'identifier des problèmes d'instabilité au lavage des premiers colorants synthétiques. Deux textiles présentaient des rouges et des orangés fugaces qui ont migré dans le tissu. Il s'agit du Ponceau RR (C.I. Acid Red 26, $n^{\circ}$ 16150) et de l'Orange II (C.I. Acid Orange $7, \mathrm{n}^{\circ} 15510$ ). Tous deux ont une faible résistance au lavage tel que démontré par l'American Association of Textile Chemists and Colorists (AATCC) qui les a testés et leur a donné une cote d'un à deux sur une échelle de cinq ${ }^{16}$.

Cette instabilité des premiers colorants a fait l'objet d'observations populaires. Alice Dubé-Lévesque décrit les problèmes rencontrés à cet égard à la fin du XIX ${ }^{\mathrm{e}}$ siècle $^{17}$ :

Je me souviens de deux couleurs qu'on achetait chez le marchand général, dans de petites boîtes rouges un peu plus grandes qu'un dé à coudre. C'était le rouge « magenta » et le violet. Il ne fallait pas trop s'y fier. Chaque lavage emportait un peu de couleur, et si vous vous tricotiez un chandail ou un châle de cette nuance, impossible de le porter sur une robe blanche.

Les premiers colorants synthétiques montraient également une instabilité à la lumière. C'est ainsi que des échantillons de violet décolorés ont été identifiés comme étant le violet de méthyle (C.I. Basic Violet 1, n 42535), dont la résistance à la lumière est cotée un sur une échelle de huit ${ }^{18}$.

L'un des textiles présentait un rose violacé qui a tourné au beige pâle. Ces fibres sont trop décolorées pour être identifiées par des tests chimiques,

16. Mary W. Ballard et al., op. cit.

17. Alice Dubé-Lévesque, Il y a soixante ans, Montréal, Fides, 1943, p. 74.

18. Mary W. Ballard et al., loc. cit. 
mais nous supposons qu'il s'agit de fuchsine ou magenta (C.I. Basic Violet $\left.14, \mathrm{n}^{\circ} 42510\right)$. Cette teinture de couleur prune se dégrade rapidement à la lumière et devient facilement de couleur beige ${ }^{19}$. C'est ainsi que nous pouvons constater un contraste important entre les couleurs naturelles qui sont restées très vives, la laque rouge et l'indigo, et les colorants synthétiques qui ont tourné au beige.

\section{Conclusion}

Les analyses ont bien démontré à quel point les colorants synthétiques ont obtenu la faveur populaire à la fin du XIX ${ }^{\mathrm{e}}$ siècle. Ces nouvelles couleurs, qui étaient faciles d'emploi, ont sans doute séduit rapidement les utilisateurs de colorants, venant ainsi modifier considérablement la gamme chromatique des textiles traditionnels. Cette démonstration, qui combine la recherche ethnologique et les tests chimiques en laboratoire, a permis de vérifier un certain savoir populaire concernant les pratiques de teinture au Québec à la fin du XIX siècle et au début du XX ${ }^{\mathrm{e}}$ siècle.

Rappelons l'intérêt d'une approche bidisciplinaire alliant les sciences pures et les sciences humaines pour l'étude de la couleur. La recherche analytique est indissociable de l'étude des sources écrites et de l'enquête ethnologique pour des résultats complets et significatifs. On ne peut plus se contenter d'un examen visuel pour identifier des fibres teintes et risquer des erreurs d'interprétation. En effet, notre perception de la couleur est déjouée par l'altération d'une teinture par la lumière, l'entretien domestique et l'usage de l'artefact. Nous oublions trop souvent qu'une même couleur peut provenir de colorants différents, avec parfois la combinaison de deux teintures différentes, ou encore qu'un même colorant peut donner plusieurs nuances.

D'une part, l'ethnologie et l'histoire apportent à la chimie des données pour documenter les colorants afin de compléter les banques d'échantillons de références qui sont essentielles aux techniques d'identification. D'autre part, les analyses chimiques permettent de compléter et de valider la documentation des collections des musées en corrigeant des erreurs d'interprétation quant à l'identification des colorants et à la datation. Ces analyses peuvent servir pour la conservation préventive des collections en identifiant des colorants plus sensibles à la lumière ou au lavage. En comprenant mieux certains problèmes de dégradation de la couleur, il nous est alors possible de mettre en pratique les mesures nécessaires pour réduire leur altération lors de la mise en réserve ou en exposition.

En terminant, rappelons que c'est grâce aux documents légués par Marius Barbeau que nous pouvons mieux comprendre les traditions de teinture de la

19. John Mills et Raymond White, The Organic Chemistry of Museum Objects, Oxford, Butterworth Heinemann, 1994 (1987), p. 153. 
fin du XIX ${ }^{\mathrm{e}}$ siècle, cette période de transition où s'opère le passage progressif des colorants naturels aux colorants synthétiques. Les enquêtes orales de Monsieur Barbeau témoignent de l'utilisation de plantes indigènes, de colorants naturels importés et de colorants synthétiques. D'autre part, l'analyse scientifique sur des artefacts des collections de musées nous apporte des renseignements supplémentaires qui sont absents de l'enquête orale et nous permettent de départager les mythes de la réalité.

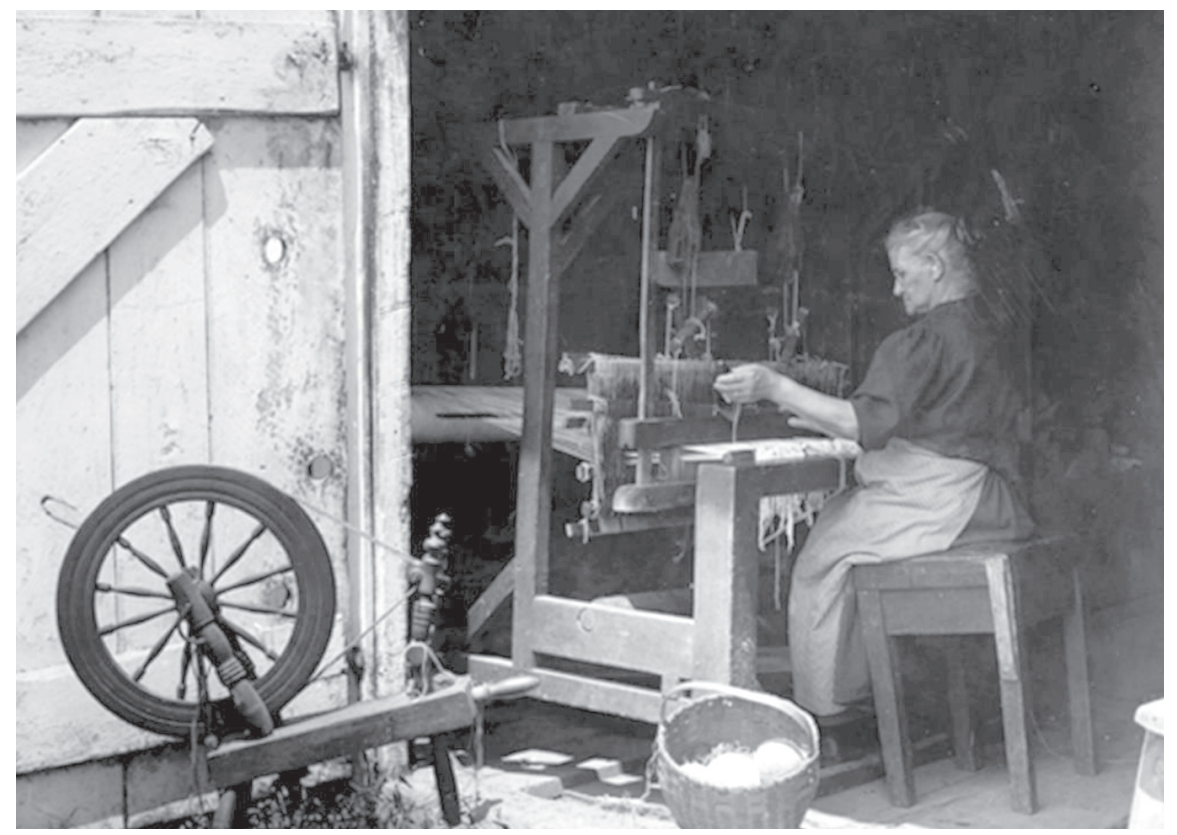

Femme tissant dans les environs de Québec, dans les années 1920.

Source : Musée canadien de l'histoire n B297-10.1. 